\title{
Propagation Characterization Based on Geographic Location Variation for 5G Small Cells
}

\author{
Byung-Jin Lee, ${ }^{1}$ Ju-Phil Cho, ${ }^{2}$ In-Ho Ra, ${ }^{3}$ and Kyung-Seok Kim ${ }^{1}$ \\ ${ }^{1}$ The Smart Radio Communication System Lab, Department of Electrical and Electronic Engineering, \\ Chungbuk National University, Cheongju, Chungbuk, Republic of Korea \\ ${ }^{2}$ Department of Radio Communication Engineering, Kunsan National University, Gunsan 573-701, Republic of Korea \\ ${ }^{3}$ School of Computer, Information and Communication Engineering, Kunsan National University, \\ Gunsan 573-701, Republic of Korea \\ Correspondence should be addressed to Kyung-Seok Kim; kseokkim@cbnu.ac.kr
}

Received 30 August 2016; Revised 22 November 2016; Accepted 15 December 2016; Published 12 January 2017

Academic Editor: Fisseha Mekuria

Copyright (C) 2017 Byung-Jin Lee et al. This is an open access article distributed under the Creative Commons Attribution License, which permits unrestricted use, distribution, and reproduction in any medium, provided the original work is properly cited.

\begin{abstract}
Developments in next-generation wireless transmission technology and efficient frequency-use research are based on understanding the characteristics of the exact radio channel. With regard to developments in next-generation mobile communication systems, performance verification of the development system is essential, for which it is necessary to estimate the exact wireless-space channel. This paper presents results of the analysis of radio propagation characteristics based on location variation in outdoor environments for small-cell 5th generation (5G) mobile systems. Changes due to variation in location were measured using a channel sounder in a microcell environment with a $0.5 \mathrm{~km}$ radius in Korea. In order to analyze the propagation characteristics, the best distribution model reflecting the characteristics of the locations was derived. A comparison between actual measurements and three-dimensional ray-tracing simulation results confirmed the validity of the measurement result.
\end{abstract}

\section{Introduction}

To meet the challenges posed by the expected rise in traffic volumes in wireless communication, research on 5th generation $(5 G)$ networks is anticipated to intensify in the next decade [1]. Rapidly rising demand for radio communication and the explosion in the number of mobile communications service subscribers have led to the need for optimization in the development of next-generation mobile communication systems [2]. The development of competitive next-generation wireless transmission technology and efficient frequency-use research are based on understanding the characteristics of the exact radio channel. In terms of developments in nextgeneration mobile communication systems, performance verification of the development system is essential, for which it is necessary to estimate the exact wireless-space channel [3]. This is because this channel is based on the exact model, including elements of the next-generation wireless transmission applications, such as frequency, time, space, and polarization [4-8].
In recent years, major national and international standardization organizations have reviewed the purposes of $5 \mathrm{G}$ mobile radio communication systems in various frequency areas, such as the $400 \mathrm{MHz}$, the $700 / 800 \mathrm{MHz}$, the $4 \mathrm{GHz}$, and the $5 \mathrm{GHz}$ bands below the $6 \mathrm{GHz}$ band. They are in the process of selecting candidate frequency bands and standardization measures based on Item 1.1 of the agenda outlined in the World Radio Communication Conference in 2015 (WRC-15) and are encouraging research participation and the development of systems all over the world [9]. The ITU-R Working Party 5D (WP 5D) proposed a candidate frequency band for IMT-advanced system applications. Moreover, private standardization organizations such as the IEEE and the 3GPP used studies on available bandwidth to present a candidate for each frequency band [10]. From the examination of the candidate frequency bands of nextgeneration mobile communications by these international standardization organizations, $3 \mathrm{GHz}$ and $4 \mathrm{GHz}$ bands have been classified as likely to be the most utilized. That involves using only part of the operators on the $3.5 \mathrm{GHz}$ band with 


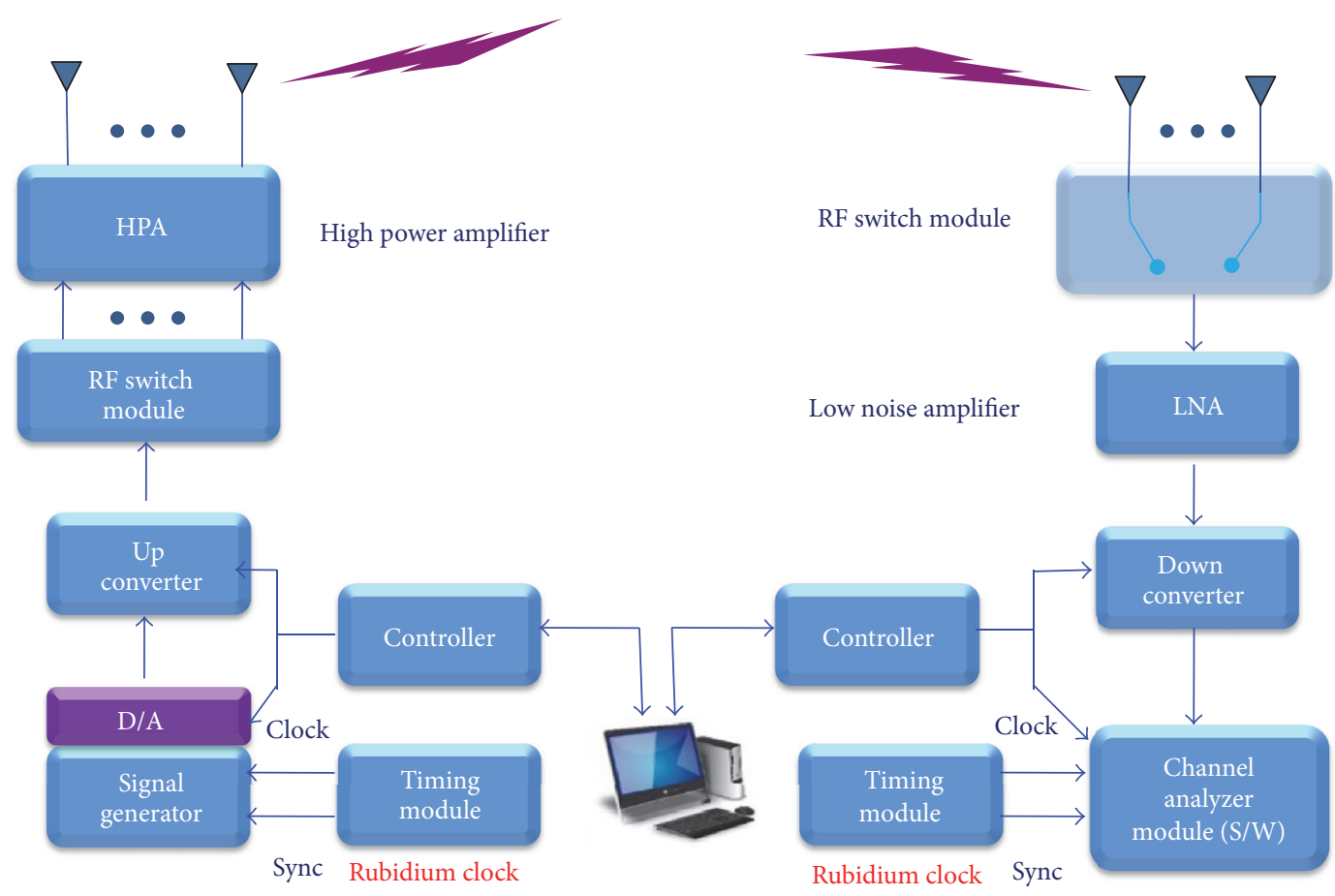

FIGURE 1: Measurement system.

LTE-TDD; particular areas, such as suburban areas, are often used for fixed wireless [11]. In countries where wireless technology is most widely used, such as the United States [12], China [13, 14], Germany [15], and Japan [16], it is necessary to take advantage of the small cell in the $3.5 \mathrm{GHz}$ band. Research has predicted that we will be able to exploit a wider area than in wide-band macro-cells.

Therefore, the $3.5 \mathrm{GHz}$ band with weak diffraction propagation characteristics is considered most likely to be used in small networks, such as small-cell and hotspot areas. This band has considerable influence over the environment through an extension of its operating frequency bandwidth, because of which it is difficult to predict the performance and operating characteristics of a system with existing characteristics analysis of channel and signal strength. Therefore, in practice, it is necessary to statistically model the characteristics of the location in accordance with changes in the environment, which are reflected in propagation channel characteristics' analysis.

Thus, mobile network operators and service providers in emerging markets are turning to small-cell solutions to meet the growing demand for voice and data traffic. The $3.5 \mathrm{GHz}$ band is under review in the context of the introduction of $5 \mathrm{G}$ systems, and radio-wave characterization research is an important field for the design, introduction, and performance evaluation of new wireless communication systems. This paper is broadly related to advances in 5G technology and measures changes in propagation characteristics according to those in the propagation environment of the given location in the $3.5 \mathrm{GHz}$ band and conducts and analyzes a raytracing simulation to verify the results. For example, to model structure such as buildings, roads, and forests, the measurements reflect the electrical properties of the constituent materials [17].

The paper is organized into five sections. Section 2 provides measurement descriptions, whereas Section 3 defines the methodology for and describes the results of a statistical analysis of the location. Section 4 is dedicated to the verification of the measurement results using the ray-tracing method. The main conclusions are listed in Section 5.

\section{Measurement Descriptions}

2.1. Measurement System. Channel impulse responses (CIR) were obtained using a channel sounder from the Electronics and Telecommunications Research Institute (ETRI) of Korea. The measurement system is specified in Table 1. Figure 1 shows the operation of the measurement system. The baseband module of the transmitter generates an intermediate frequency (IF) signal with a $100 \mathrm{MHz}$ bandwidth. The radio frequency (RF) module has eight switching signals, and the adjacent high-power amplifier (HPA) module that follows has a maximum power of up to $33 \mathrm{dBm}$. The samples stored by the sounder are I and Q data forms saved at one datum per second. A pseudorandom sequence of length 4096 was continuously generated at the transmitter (Tx). The Tx and receiver $(\mathrm{Rx})$ were capable of recording the measurement position via a built-in GPS. At the $\mathrm{Rx}$, CIRs were obtained by slide, correlating the received signal with a synchronized copy of the sequence. The central frequency was $3.5 \mathrm{GHz}$. The number of antennas in each piece of the transmitter and receiver equipment was eight. The $\mathrm{Rx}$ was located at the end 
TABLE 1: The main parameters of measurement system.

\begin{tabular}{|c|c|}
\hline Center frequency & $3.5 \mathrm{GHz}$ \\
\hline Bandwidth & $100 \mathrm{MHz}$ \\
\hline Transmission power & $33 \mathrm{dBm}$ \\
\hline Transmission signal & $4096 \mathrm{PN}$ sequence \\
\hline Sampling rate & $\begin{array}{c}200 \text { Msamples/s } \\
\text { (with } 2 \text { oversampling } \\
\text { processes) }\end{array}$ \\
\hline Tx antenna \& Rx antenna & $\begin{array}{l}\text { Omnidirectional } \\
\text { (with } 5.22 \mathrm{dBi} \text { ) }\end{array}$ \\
\hline Tx antenna height & $7.3 \mathrm{~m}$ \\
\hline $\mathrm{Rx}$ antenna height & $2 \mathrm{~m}$ \\
\hline Number of antennas & 8 \\
\hline $\begin{array}{l}\text { Average speed of measurement } \\
\text { car }\end{array}$ & $40 \mathrm{~km} / \mathrm{h}$ \\
\hline
\end{tabular}

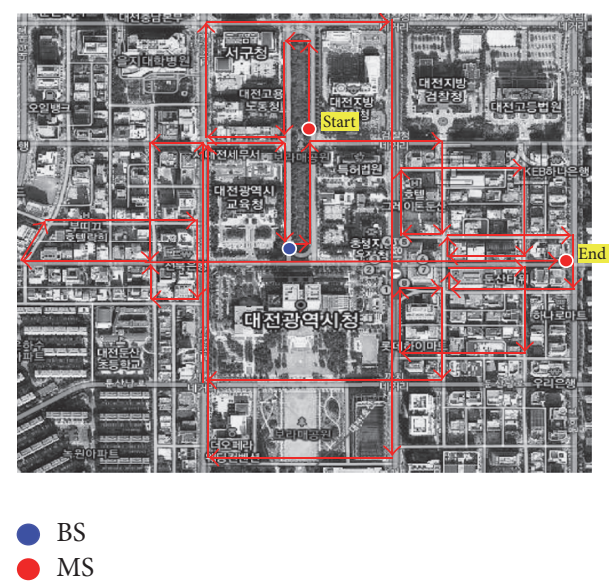

FIgURE 2: Scenario for the analysis of location characteristics.

of the roof of a vehicle, and the antenna was $2 \mathrm{~m}$ high. The Tx was mounted on a pole at a height of $7.3 \mathrm{~m}$ to simulate typical small-cell deployment on the street.

2.2. Measurement Planning and Environment. Most prevalent propagation models are line-of-sight (LOS) and non-lineof-sight (NLOS) propagation prediction models for pointto-point scenarios [18]. However, since a real NLOS environment does not offer the possibility of a shadow area (where signals are not transmitted), radio-wave-property prediction is challenging. In particular, even if the base station is installed, the predictable area for the transfer of a signal is limited by tall building structures in urban areas. Moreover, depending on the location of the buildings, the size of the population, and vehicular traffic, changes in the radio wave can be very large [19]. However, little, if any, prediction is available for this part, which is also reflected in the propagation model. Therefore, following the assessment of a radio-wave measuring system in a real environment, it is necessary to reflect the derived location probability distribution in the propagation model.

The measurement location was near Daejeon City Hall in the Republic of Korea, a typical complex urban environment. The measurement scenario consisted of two Tx locations and $\mathrm{Rx}$ routes. Figure 2 shows an aerial map of the measurement area. In Figure 2, the Tx location is fixed on site, with the Rx driven along the measurement routes. This was intended to analyze the variations in signal strength due to differences between the topography and the environment. The measurement range varied from a $20 \mathrm{~m}$ radius to $500 \mathrm{~m}$ radius from the Tx. Small outdoor cells are expected to support mediumto-high mobility (up to $50 \mathrm{~km} / \mathrm{h}$ ) while maintaining good quality of service [20]. The average moving velocity of the measuring vehicle was $40 \mathrm{~km} / \mathrm{h}$. The measuring time was up to one hour, and the total number of measurement points was 1,420 .

\section{Statistical Analysis of Location and Results}

3.1. Analytical Methodologies. Location percentage refers to a statistical representation of the variation in signal strength on a wireless channel due to the change in terrain or environment. Variation in signal strength was obtained according to the location percentage for a given unit area. A parameter-deriving method considering location percentage is a reference in ITU-R p. 1411 [21]. Procedures for statistically modeling the change in location characteristics are shown in Figure 3.

First, the data obtained from the measured field were classified into LOS and NLOS regions to derive their respective path-loss values. The variations in the signal were then analyzed based on the fitness of the measured signal values to derive a location correction factor. Following this, the calculation of LOS distance according to location percentage allowed for that of path loss. Finally, a path-loss graph was derived per location percentage.

3.2. Median Model for Path Loss. Note that distance is not travelled distance but refers to the maximum radius. There are points drawn for any generally given distance, corresponding to the change in location due to the movement of the measuring vehicle. Figure 4 shows path loss in accordance with received distance. As distance increases, the value of path loss significantly distributes due to location variation. This offers a clear picture of the short-range LOS and longrange NLOS regions. The two regions are separated by a sharp attenuation, with a significant increase in loss. The path-loss value, which changes according to distance, appears to be along the following distribution [22]:

$$
\mathrm{PL}(d)=L_{0}+10 n \log _{10}\left(\frac{d}{d_{\text {ref }}}\right)+X_{\sigma},
$$

where $L_{0}$ is the initial PL value and $n$ is the PL index. These were estimated by a regression analysis of the measured reception data. $d_{\text {ref }}$ is $20 \mathrm{~m} . X_{\sigma}$ is the standard deviation (STD). The parameters derived in each region are given in Table 2. 
TABLE 2: Locations characteristics derivation parameters.

\begin{tabular}{lccccrr}
\hline & \multicolumn{3}{c}{ Measurement conditions } & & \multicolumn{2}{c}{ PL } \\
Area & $f(\mathrm{GHz})$ & $h_{b}(\mathrm{~m})$ & $h_{m}(\mathrm{~m})$ & $d_{\text {ref }}(\mathrm{m})$ & $L_{0}$ & $X_{\sigma}$ \\
\hline LOS region & \multirow{2}{*}{3.5} & 7.3 & 2.0 & 20 & -15.05 & 1.63 \\
NLOS region & & & & & -19.59 & 4.79 \\
\hline
\end{tabular}

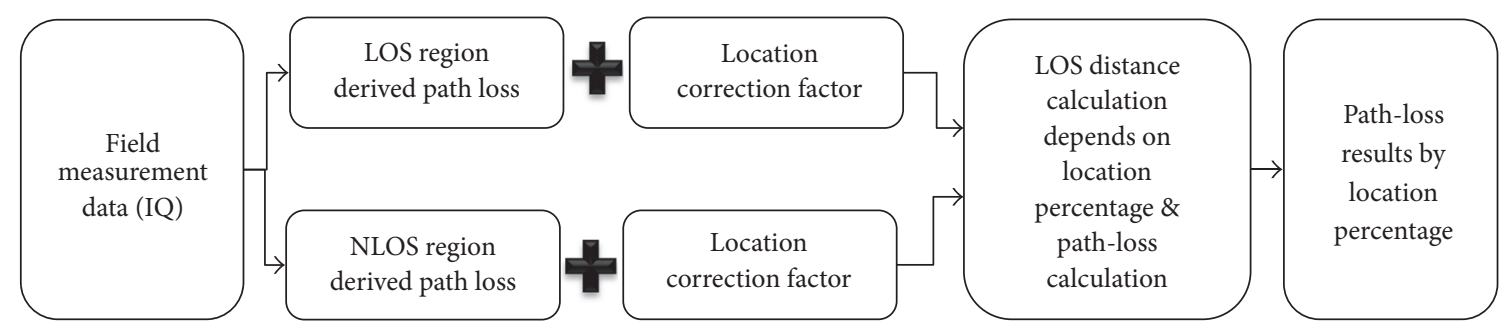

FIgURE 3: Steps for the derivation of location characteristics.

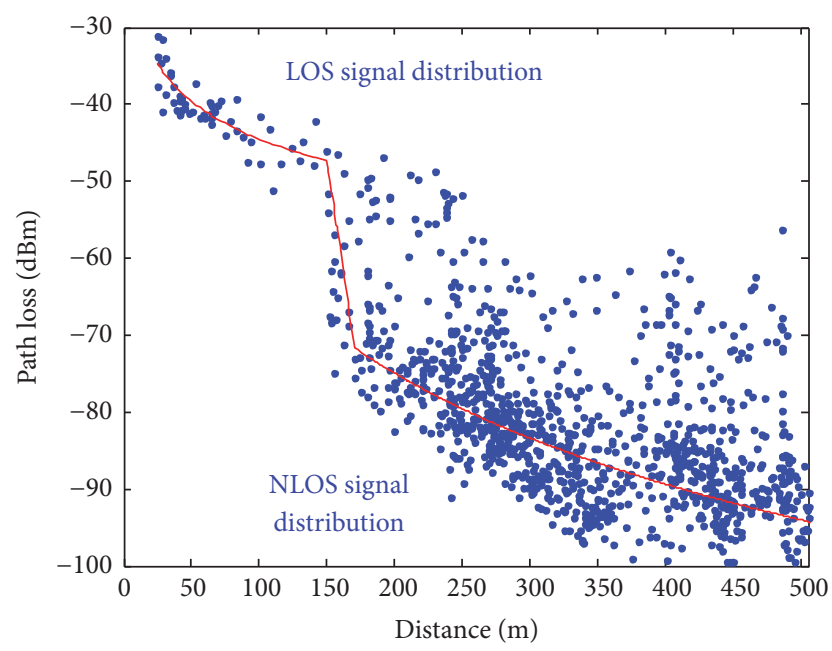

FIGURE 4: Measured path loss plotted against distance from the transmitter.

3.3. Best-Fit Distribution from Location Variation. Location variation was quantified by calculating the standard deviation of the data relative to the median fit line. The location correction factor was intended to determine the best-fit distribution represented as a probability density function (PDF) based on the variation of the signal.

In Figure 5, the probability density function of the difference between the median fit and the measured received data is shown for the best-fit distribution. The values depicted in Figure 5 provide an idea of the variation in the received signal over the course of the measurement. As a result of the examined PDFs, it seemed reasonable to consider the LOS and NLOS models of location variation as an extreme value distribution and a logistic distribution, respectively. The LOS region might exhibit some deviation in a small number of data items compared to the NLOS region. Sometimes, large improvements in the values are obtained because proximity to a transmitter causes antenna pattern issues and calibration issues. In the measurement environment, an extreme value distribution is a better fit than a logistic distribution because it is similar to the trend in signal variation. More specifically, the correction to be added to the median value of the LOS and NLOS path loss is a function of location percentage given by

$$
\begin{aligned}
\Delta L_{\text {extreme }}(p) & =E^{-1}\left(\frac{p}{100}\right) \sigma, \\
\Delta L_{\text {logistic }}(p) & =\operatorname{Lo}^{-1}\left(\frac{p}{100}\right) \sigma,
\end{aligned}
$$

where $\sigma$ is the standard deviation (LOS was $9.65 \mathrm{~dB}$ and NLOS was $10.83 \mathrm{~dB}), E^{-1}(\cdot)$ is the inverse-extreme-value cumulative distribution function (CDF) [23], and $\mathrm{Lo}^{-1}(\cdot)$ is the inverse logistic cumulative distribution [24]. The value of the correction factor corresponding to each percentage is shown in Figure 6. As seen in the figure, the CDF curve-slope of each correction coefficient represents the effect of location for each percentage value. If the curve is perfectly vertical, it has the same location correction factor; the location correction factor in these cases is null. The longer the slope, the greater the impact due to variation in location.

3.4. LOS Distance Derivation. Calculating the distribution of LOS and NLOS transition distance according to location percentage is a step in calculating path loss. This was investigated while expanding the radius of the $\mathrm{Rx}$ at the basis of the transmitter. If the receiver was outside a building, it was classified as LOS or NLOS by examining the direct line between Tx and Rx for building blockage. The LOS distance exceeded for a given percentage of the locations is shown in Figure 7. The resulting data were assigned to $15 \%$ of the locations for a distance of $0.5 \mathrm{~km}$. The model predicted that $100 \%$ of the paths were LOS $36 \mathrm{~m}$ from the Tx. The LOS 


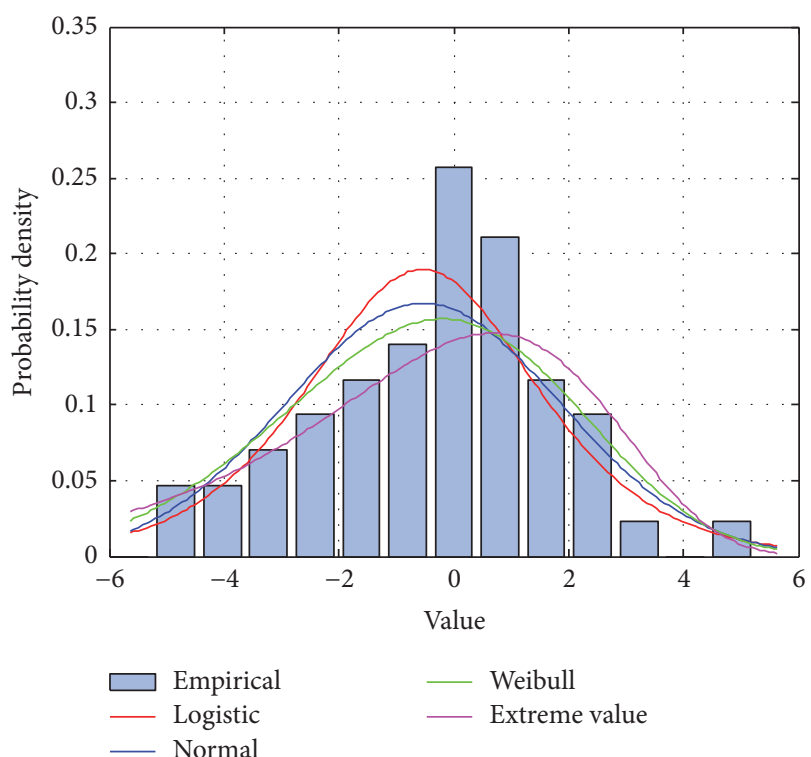

(a) LOS region

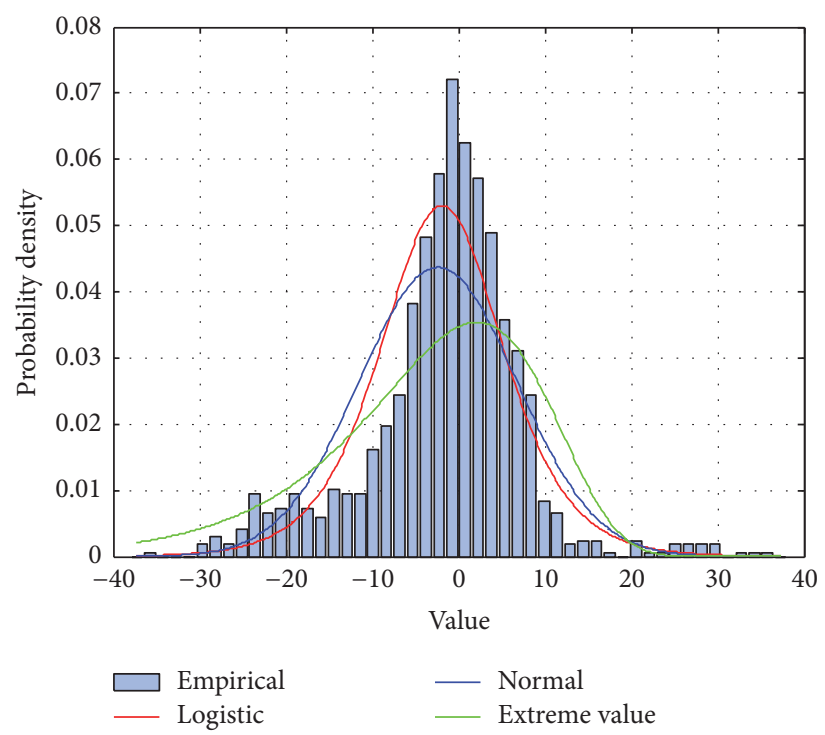

(b) NLOS region

FIGURE 5: Probability density functions of location variation.

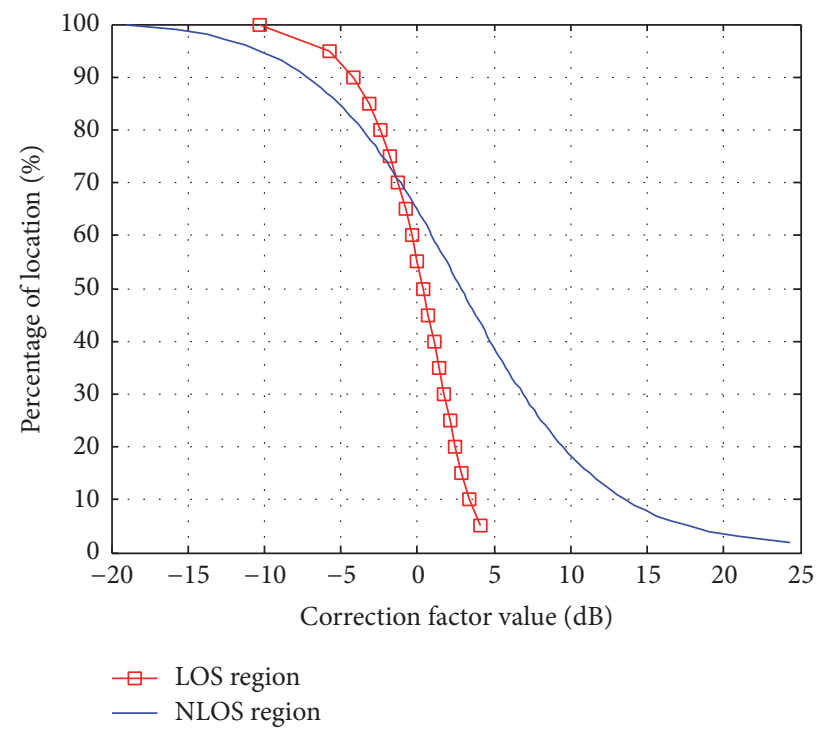

FIGURE 6: The correction factor values corresponding to the respective percentages.

distance $d_{\text {LOS }}$ exceeded for a given percentage of locations was given by

$$
\begin{aligned}
& d_{\operatorname{LOS}}(p) \\
& = \begin{cases}4514.4 e^{-0.172(p / 100)}+817.1 e^{-0.001(p / 100)}-593.3, & p<85 \\
170.51-119.51\left(\frac{p}{100}\right), & \text { otherwise }\end{cases}
\end{aligned}
$$

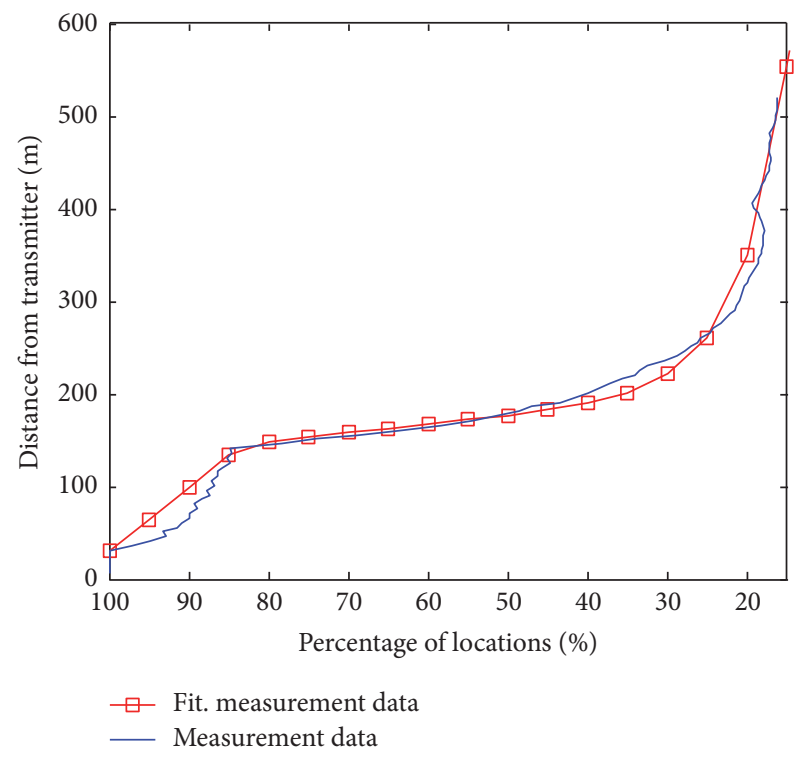

FIGURE 7: LOS distance exceeded for a given percentage of locations.

with $d_{\mathrm{LOS}}$ in meters; $p$ is the location percentage (15\% 100\%). The path loss at distance $d$ can then be given by

$$
\begin{aligned}
& \mathrm{PL}(p) \\
& = \begin{cases}\mathrm{PL}_{\mathrm{LOS}}, & d<d_{\mathrm{LOS}} \\
\mathrm{PL}_{\mathrm{NLOS}}, & d>d_{\mathrm{LOS}}+w . \\
\mathrm{PL}_{\mathrm{LOS}}+\frac{\left(\mathrm{PL}_{\mathrm{NLOS}}-\mathrm{PL}_{\mathrm{LOS}}\right)\left(d-d_{\mathrm{LOS}}\right)}{w}, & \text { otherwise }\end{cases}
\end{aligned}
$$

$w$ is used to provide a transition region between the LOS and NLOS regions and typically has a width of $w=20 \mathrm{~m}$. 


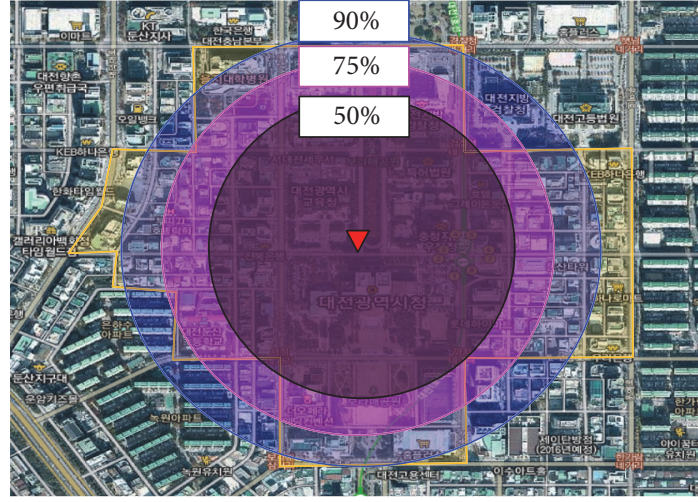

Figure 8: Ratio of the unit area for each location percentage.

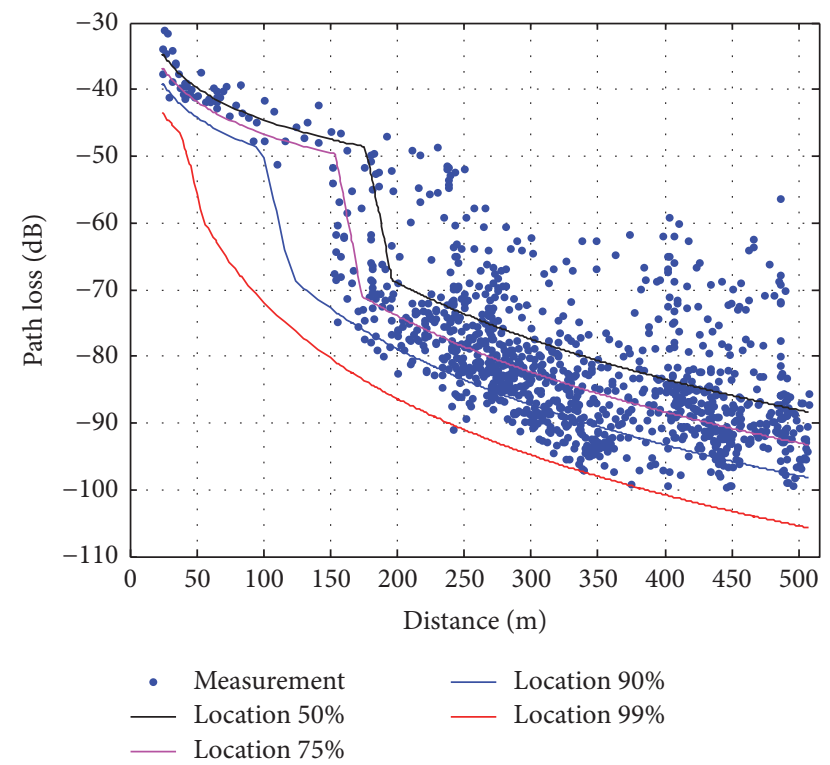

Figure 9: Path loss for each location percentage.

3.5. Path-Loss Result according to Location Percentage. Figure 8 shows the terrain ratio of the unit area according to location percentage. The range for a location percentage of $50 \%$ was a radius of $255 \mathrm{~m}$ from the transmitter; at $75 \%$, it was $383 \mathrm{~m}$; at $90 \%$, it was $459 \mathrm{~m}$.

Figure 9 shows path loss according to location percentage and highlights the result obtained by applying location percentage based on the measured data. The blue dots represent measured data, and each line shows the result obtained by applying location percentages of 50\%, 75\%, 90\%, and $99 \%$. This loss increases with increasing distance and, starting at approximately $160 \mathrm{~m}$, rapidly increases. This is the attenuation of the signal due to the corner distance, $L_{\text {corner }}$, which includes the statistics of location variation in the LOS and NLOS regions, and provides a statistical model for the corner distance between the LOS and NLOS regions. $L_{\text {corner }}$ was given as $20 \mathrm{~dB}$ in an urban area. Assuming a 50\% location percentage, the LOS area based on a radius of $255 \mathrm{~m}$ from the transmitter was up to $176 \mathrm{~m}$. Assuming a $90 \%$ location

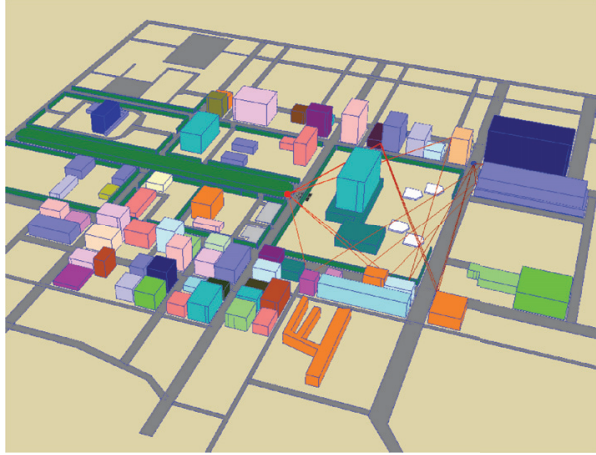

FiguRE 10: Ray-tracing simulation for a 50\% location percentage.

percentage, the LOS area based on a radius of $459 \mathrm{~m}$ from the transmitter was up to $99 \mathrm{~m}$. When comparing the path-loss values of $50 \%$ and $90 \%$ of the location percentage at $150 \mathrm{~m}$, there was a difference of approximately $-25.45 \mathrm{~dB}$ in each $(-47.5 \mathrm{~dB}$ and $-72.95 \mathrm{~dB})$.

Thus, the results of path loss indicated a difference in response to spatial variation, such as distance and building density. Therefore, it is necessary to statistically model the change in channel characteristics due to those in the surrounding environment.

\section{Verification of Measurement Results Using the Ray-Tracing Method}

To verify the effectiveness of the measurement results, a simulation condition was created based on a geographic information system map in a real measurement environment. The simulation environment, including buildings and forest information, was configured similarly to the real environment. The height of the building, the width of the road, the density of the building, and the materials (asphalt, metal, cement, wood, etc.) were given different values for reliability, where permittivity, conductivity, and transmission of each material were considered (e.g., the permittivity of wood is 1.99 , its conductivity is 0.012 , and it does not transmit electricity). The height of the $\mathrm{Rx}$ antenna was $2 \mathrm{~m}$ and that of the Tx was $7.3 \mathrm{~m}$. The 3D simulation was conducted by adding information concerning radio waves, such as diffraction, reflection, and scattering [25].

Figures 10 and 12 show a ray-tracing screen designed by considering location percentages of $50 \%$ and $90 \%$ based on the actual measurement environment. The simulation environment with a location percentage of $90 \%$ showed greater building density and configuration than that of $50 \%$. The simulation results are shown in Figures 11 and 13 for comparison with the measurement results. The blue dots show the results of the ray-tracing simulation, and the circled red line shows the result of applying a ray-tracing simulation with location percentages of $50 \%$ and $90 \%$. This was applied in the same way as the analysis method in Section 3. The black line shows actual measurement results for location percentages of $50 \%$ and $90 \%$. 


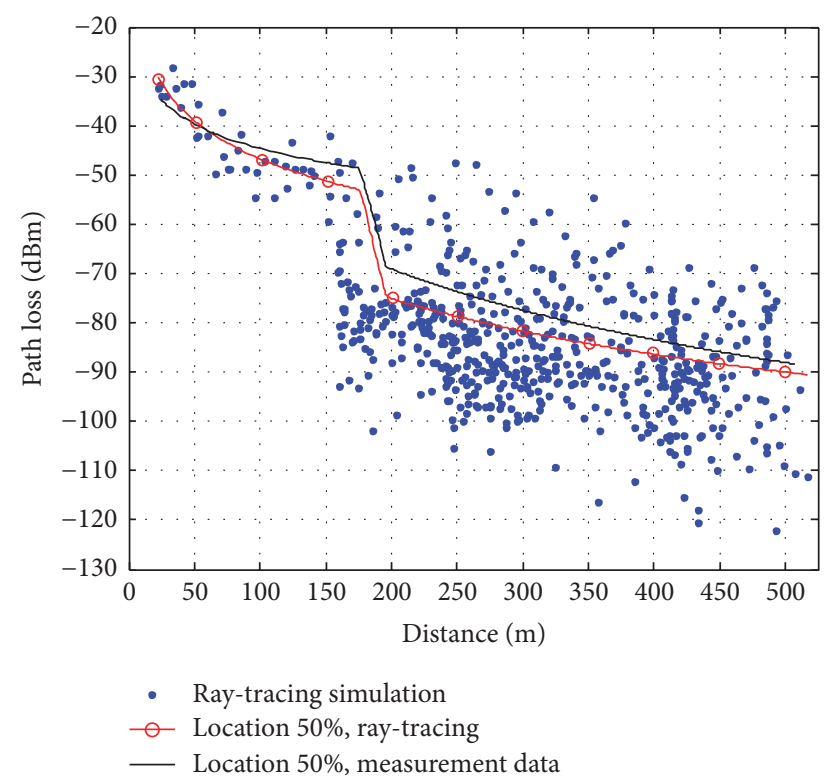

FIGURE 11: Comparing measurement results with ray-tracing results for a $50 \%$ location percentage.

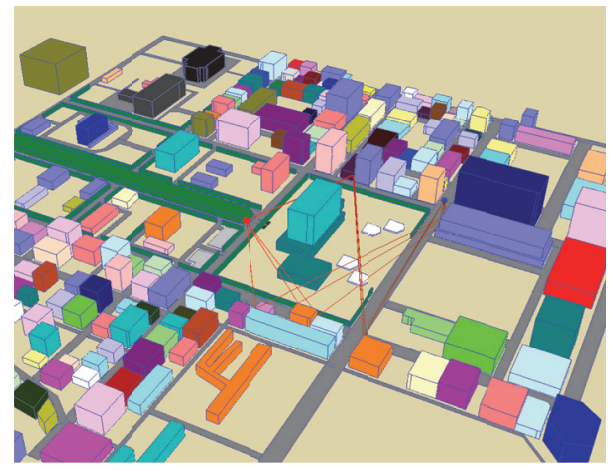

FIGURE 12: Ray-tracing simulation for a $90 \%$ location percentage.

The ray-tracing results were slightly different from the measurement results, but the differences in trend were very weak. Considering that the overall trend was a reduction, it was similar to the difference with $10 \mathrm{~dB}$. Therefore, the measurement data in this paper made it possible to determine reliability.

\section{Conclusion}

Major national and international standardization organizations have recently been considering the use of 5 th generation (5G) mobile radio communication systems in a variety of frequency domains. Of these, the $3 \mathrm{GHz}$ and $4 \mathrm{GHz}$ bands are classified as likely to be the most used. The bands are likely to be used in small networks at present, such as small-cell and hotspot areas, and should statistically model the characteristics of a given location because changes in

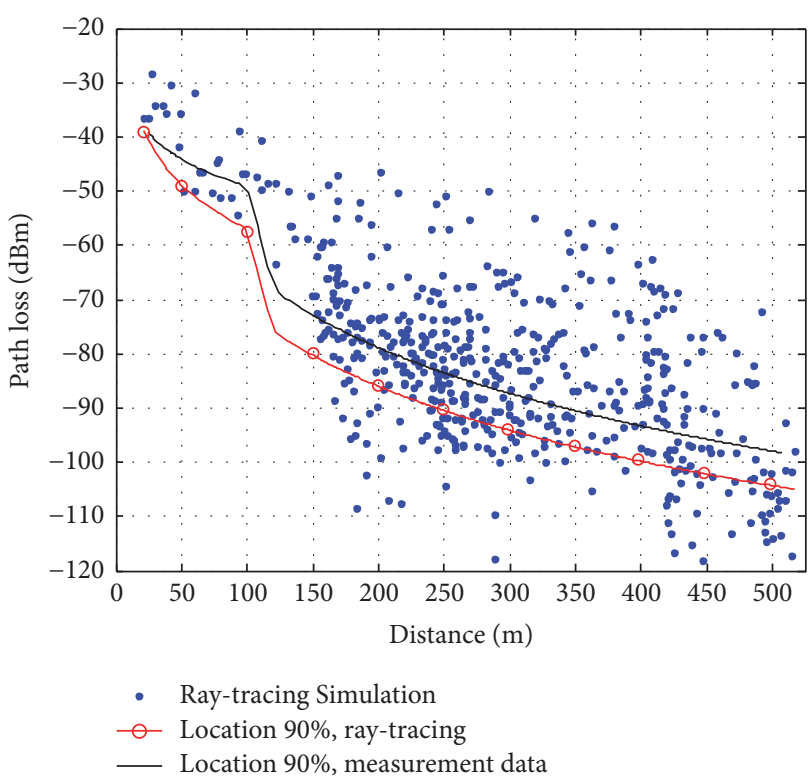

FIGURE 13: Comparing measurement results to ray-tracing results for a $90 \%$ location percentage.

environment must be reflected in the prediction and analysis of radio channel characteristics.

For this reason, this paper measured changes in propagation characteristics according to variation in the propagation environment of locations in the $3.5 \mathrm{GHz}$ band in Korea. The best statistical distribution to characterize the received signal location behavior was proposed, and a set of critical parameter values for this distribution were calculated from the measurement data. In the analysis of location percentage, the logistic was first found to be the best-fit distribution for traffic-affected NLOS location records, whereas the extreme value was found to be the best fit for LOS location points. This means that the operation of the reception signal in an urban environment was highly dependent on building density and traffic, based on the measured position, and these factors can lead to a higher standard deviation in an urban environment. We also enhanced the reliability of the simulation by applying a simulation environment similar to the actual measured environment. Comparing the measurement results with $3 \mathrm{D}$ ray-tracing simulation results proved the validity of the measurement results.

The location variation-characteristics studied in this paper required predicting the transfer of stable signals from a hotspot area, in order for the number of users to increase exponentially. This prediction error is expected to be significantly reduced and will help in cell planning for nextgeneration mobile communication services on the $3.5 \mathrm{GHz}$ band.

\section{Competing Interests}

The authors have no conflict of interests to declare regarding the publication of this paper. 


\section{Acknowledgments}

This work was supported by the National Foundation of Korea (NSF) grant funded by the Korean Government (MSIP) (no. 2016R1A2B40130002).

\section{References}

[1] T. Taleb, "Toward carrier cloud: potential, challenges, and solutions," IEEE Wireless Communications, vol. 21, no. 3, pp. 80-91, 2014.

[2] Cisco Visual Networking Index, "Global mobile data traffic forecast update, 2011-2016," White Paper, 2012.

[3] A. Osseiran, F. Boccardi, V. Braun et al., "Scenarios for 5G mobile and wireless communications: the vision of the METIS project," IEEE Communications Magazine, vol. 52, no. 5, pp. 2635, 2014.

[4] M. Landmann, K. Sivasondhivat, J.-I. Takada, I. Ida, and R. Thomä, "Polarization behavior of discrete multipath and diffuse scattering in urban environments at $4.5 \mathrm{GHz}$," Eurasip Journal on Wireless Communications and Networking, vol. 2007, Article ID 57980, 2007.

[5] V. Degli-Esposti, D. Guiducci, A. de'Marsi, P. Azzi, and F. Fuschini, "An advanced field prediction model including diffuse scattering," IEEE Transactions on Antennas and Propagation, vol. 52, no. 7, pp. 1717-1728, 2004.

[6] J. Poutanen, J. Salmi, K. Haneda, V.-M. Kolmonen, F. Tufvesson, and P. Vainikainen, "Propagation characteristics of dense multipath components," IEEE Antennas and Wireless Propagation Letters, vol. 9, pp. 791-794, 2010.

[7] J. Järveläinen and K. Haneda, "Sixty gigahertz indoor radio wave propagation prediction method based on full scattering model," Radio Science, vol. 49, no. 4, pp. 293-305, 2014.

[8] C. Jansen, S. Priebe, C. Moller et al., "Diffuse scattering from rough surfaces in $\mathrm{THz}$ communication channels," IEEE Transactions on Terahertz Science and Technology, vol. 1, no. 2, pp. 462-472, 2011.

[9] ITU-R preparatory studies for World Radio Communication Conference 2015 (WRC-15), http://www.itu.int/pub/R-ACTWRC.12-2015/en.

[10] ETSI TS 136101 (V12.9.0) (10/2015): “LTE: Evolved Universal Terrestrial Radio Access (E-UTRA); User Equipment (UE) radio transmission and reception (3GPP TS 36.101 version 12.9.0 Release 12)".

[11] http://gsacom.com/paper/lte-tdd-td-lte-global-status-71-commercially-launched-networks/.

[12] NTIA, "An Assessment of the Near-term Viability of Accommodating Wireless Broadband Systems in the $1675 \sim 1710 \mathrm{MHz}$, $1755 \sim 1780 \mathrm{MHz}, 3500 \sim 3650 \mathrm{MHz}$, and 4200 4220 MHz, 4380 $4400 \mathrm{MHz}$ Bands," U.S Department of Commerce, 2010, https:// www.ntia.doc.gov/files/ntia/publications/fasttrackevaluation 11152010.pdf.

[13] APT, "Frequency usage of the band 3400 3600 MHz," in Proceedings of the 14th APT Wireless Group Meeting, APT, Bangkok, Thailand, 2013, http://www.apt.int.

[14] Huawei, “3.5GHzffkfhLTE-Advanced," 2012, http://www.soumu .go.jp/.

[15] ECC, "Preferred frequency arrangement for the 3400 3600 MHz band in ECC Decision (11)06," ECC PT1 (13)023, 2013.

[16] MIC, Announcement of Frequency Reorganization Action Plan, 2013.
[17] F. Fuschini, H. El-Sallabi, V. Degli-Esposti, L. Vuokko, D. Guiducci, and P. Vainikainen, "Analysis of multipath propagation in urban environment through multidimensional measurements and advanced ray tracing simulation," IEEE Transactions on Antennas and Propagation, vol. 56, no. 3, pp. 848-857, 2008.

[18] P. Almers, E. Bonek, A. Burr et al., "Survey of channel and radio propagation models for wireless MIMO systems," EURASIP Journal on Wireless Communications and Networking, vol. 2007, Article ID 019070, 2007.

[19] T. Imai, "Performance evaluation method for propagation loss prediction model based on wavelet analysis," IEICE Transactions on Communications, vol. 95, no. 10, pp. 1335-1343, 2012.

[20] A. Osseiran, F. Boccardi, V. Braun et al., "The METIS 2020 Project-Laying the foundation of 5G," METIS 2020, Novmber 2012, https://www.metis2020.com/.

[21] ITU, "Propagation data and prediction methods for the planning of short-range outdoor radio communication systems and radio local area networks in the frequency range $300 \mathrm{MHz}$ to 100 GHz," ITU-R P 1411-7, 2013.

[22] H.-S. Jo and J.-G. Yook, "Path loss characteristics for IMTadvanced systems in residential and street environments," IEEE Antennas and Wireless Propagation Letters, vol. 9, pp. 867-871, 2010.

[23] S. Coles, An Introduction to Statistical Modeling of Extreme Values, Springer Series in Statistics, Springer, London, UK, 2001.

[24] W. G. Stock, "On relevance distributions," Journal of the American Society for Information Science and Technology, vol. 57, no. 8, pp. 1126-1129, 2006.

[25] Y. Corre and Y. Lostanlen, “Three-dimensional urban EM wave propagation model for radio network planning and optimization over large areas," IEEE Transactions on Vehicular Technology, vol. 58, no. 7, pp. 3112-3123, 2009. 

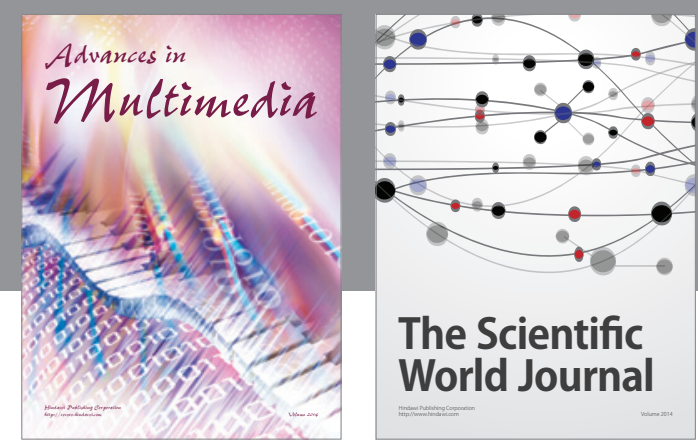

The Scientific World Journal
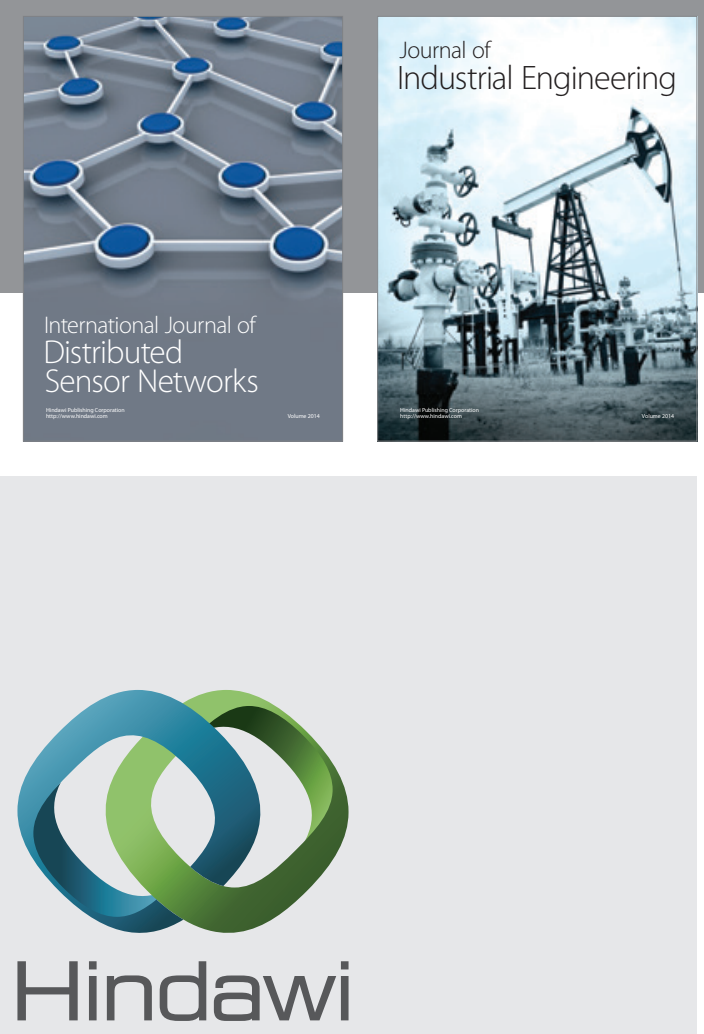

Submit your manuscripts at

https://www.hindawi.com

\section{Computer Networks} and Communications
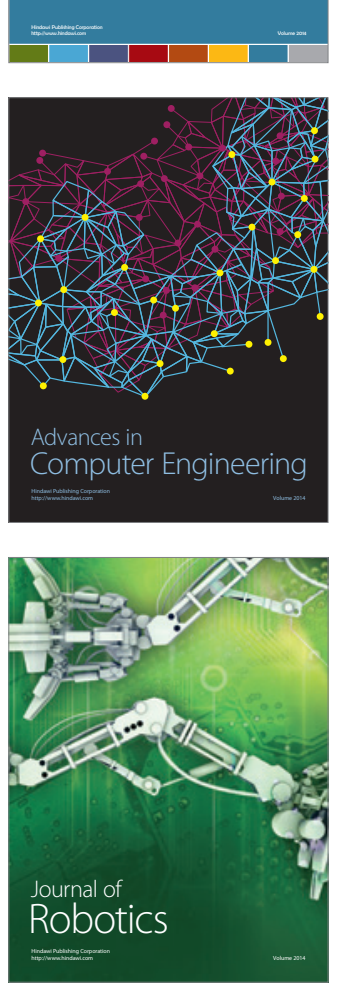
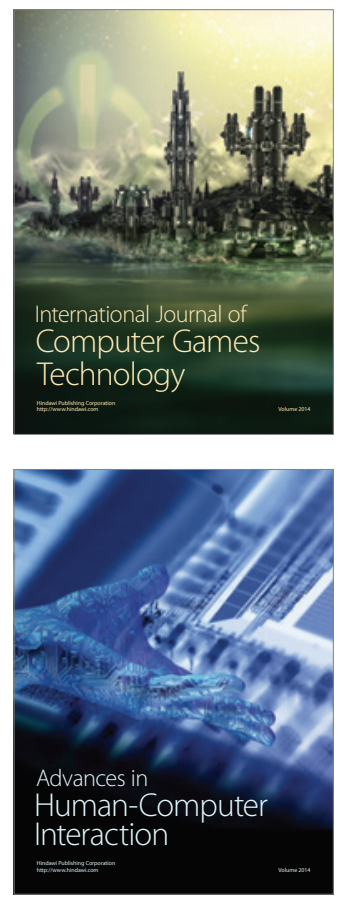
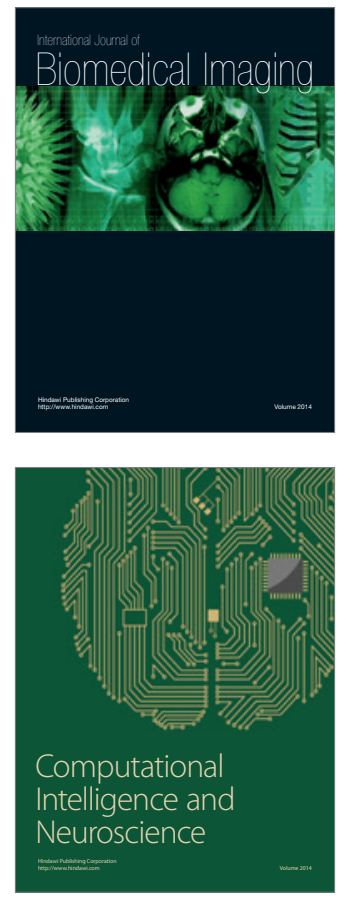
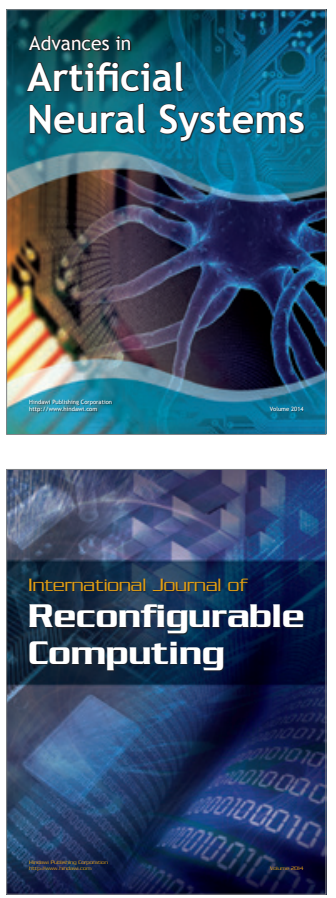
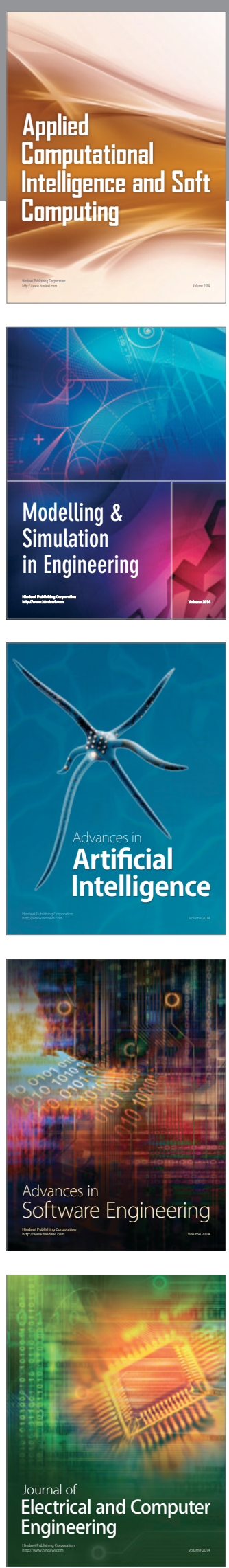